Characteristics of Divertor Heat and Particle Deposition with Intrinsic and Applied 3-D Fields in NSTX H-mode Plasmas

J. W. Ahn, J. Canik, R. Maingi, T. K. Grey, J. D. Lore, A. G. McLean, J. Park, A. L. Roquemore, V. A. Soukhanovskii

June 7, 2010

Journal of Nuclear Materials 
This document was prepared as an account of work sponsored by an agency of the United States government. Neither the United States government nor Lawrence Livermore National Security, LLC, nor any of their employees makes any warranty, expressed or implied, or assumes any legal liability or responsibility for the accuracy, completeness, or usefulness of any information, apparatus, product, or process disclosed, or represents that its use would not infringe privately owned rights. Reference herein to any specific commercial product, process, or service by trade name, trademark, manufacturer, or otherwise does not necessarily constitute or imply its endorsement, recommendation, or favoring by the United States government or Lawrence Livermore National Security, LLC. The views and opinions of authors expressed herein do not necessarily state or reflect those of the United States government or Lawrence Livermore National Security, LLC, and shall not be used for advertising or product endorsement purposes. 


\title{
Characteristics of Divertor Heat and Particle Deposition with Intrinsic and Applied 3-D Fields in NSTX H-mode Plasmas
}

\author{
J-W. Ahn ${ }^{1}$, J. M. Canik ${ }^{1}$, R. Maingi ${ }^{1}$, T. K. Gray ${ }^{1}$, J. D. Lore ${ }^{1}$, A. G. McLean ${ }^{1}$, J.-K. Park ${ }^{2}$, \\ A. L. Roquemore ${ }^{2}$, and V. A. Soukhanovskii ${ }^{3}$ \\ ${ }^{1}$ Oak Ridge National Laboratory, Bethel Valley Road, Oak Ridge, TN 37831, USA \\ ${ }^{2}$ Princeton Plasma Physics Laboratory, Princeton, NJ 08543, USA \\ ${ }^{3}$ Lawrence Livermore National Laboratory, 7000 East Ave., Livermore, CA 94551, USA
}

\begin{abstract}
Divertor heat and particle flux profiles are modified by externally imposed non-axisymmetric magnetic perturbations in the National Spherical Torus Experiment. The applied 3-D field causes strike point splitting that is represented as local peaks and valleys in the divertor profiles. The plasma response was included in the field line tracing by taking account of the B-field generated by the plasma current up to a certain fraction of normalized flux inside the separatrix and being superposed to the vacuum field. The inclusion of plasma response does not significantly affect the location and spacing of the split strike points at the divertor surface. A modest level of divertor profile modification is found to occur even without the application of 3-D fields in certain high triangularity $(\delta=0.65-0.8)$ discharges, with the location of local peaks and valleys same before and after the application. The intrinsic error field from the non-circularity of PF5 coil is known to have primarily $n=3$ component in NSTX and was modelled to be included in the vacuum field line tracing. The produced puncture plot of the field line along with the connection length profile shows that the radial location of local peaks agrees well with the measurement, identifying intrinsic error field as a possible source of intrinsic strike point splitting. The radial location of local peaks in the profiles during the triggered ELM by the applied $n=3$ field is the same before and after the 3-D field application. This shows that the heat flux from the triggered ELMs appears to follow the imposed $n=3$ field structure.
\end{abstract}

\section{Introduction}

Tokamaks are commonly regarded to have toroidally axisymmetric magnetic configuration. Therefore, plasma facing components (PFCs) are also designed and built axisymmetric to protect areas where high heat and particle fluxes are expected from the 2-D

*Corresponding author's contact information: jahn@pppl.gov, Princeton Plasma Physics Laboratory, MS-15, Princeton, NJ 08543, USA 
equilibrium. However, the application of small, non-axisymmetric magnetic field perturbations produced by in-vessel or ex-vessel coils has been recently found to modify and break the axisymmetry of divertor profiles as well as to have significant impact on the Edge Localized Mode (ELM) characteristics in tokamaks [1]. As the International Thermonuclear Experimental Reactor (ITER, "The Way" in Latin) presently considers the use of 3-D magnetic perturbation for the ELM suppression as a possible option [2], the effect of these 3-D fields on the heat and particle footprints on the divertor plates is of substantial interest and has recently drawn attention from various machines $[3,4,5,6]$.

In DIII-D [7], large type-I ELMs have been successfully eliminated [8, 9] by applying resonant magnetic perturbations (RMPs) produced by a series of coils inside the vacuum vessel (internal or "I-coils"). In the National Spherical Torus Experiment (NSTX) [10], long (> $600 \mathrm{~ms}$ ) ELM-free H-mode plasmas were achieved by heavy lithium evaporation and coating onto the plasma facing components [11]. Application of 3-D fields to these plasmas triggered ELMs with the ELM frequency controlled by the frequency of applied 3-D field coil currents in case a square wave coil current was applied $[12,13]$, while the ELM frequency tend to increase with the increasing coil current in case of constant coil current [13]. Note that the data presented in this paper were obtained with constant coil current. When the external 3-D field is applied, modification of the magnetic equilibrium produces a 3-D structure of perturbed magnetic field lines in the plasma edge, where the poloidal magnetic flux is re-organized into topological structures known as homoclinic tangles [14]. Perturbed by non-axisymmetric 3-D fields, the separatrix is split into multiple invariant manifolds forming a 3-D lobe structure for the open field lines (in other words, field lines that hit the material surface), which are a mixture of long connection length stochastic field lines and short connection length laminar field lines. The lobe structure of the open field lines generates a striated, ie split, strike point (SP) pattern radially across the divertor target surface. This structure is expected to be reflected in the measured divertor heat and particle flux profiles and such an observation during the 3-D perturbation field application was recently reported in DIII-D [1, 14, 15] and NSTX [16] H-mode plasmas.

In addition to the explicit application of 3-D fields by coils, non-axisymmetric magnetic perturbations can be also produced from other sources: intrinsic error fields; internal MHD modes; toroidal field ripples, etc. These 3-D fields can in principle make a similar impact on the plasma to the explicitly imposed 3-D fields described above. In fact, SP splitting of heat and particle flux profiles has been observed even without explicit application of 3-D fields in certain high-triangularity $(\delta=0.65-0.8)$ discharges in NSTX [16]. This SP splitting appears to be related to the intrinsic error fields at least to a certain extent. We will therefore call the SP splitting without explicit application of 3-D fields the, intrinsic SP splittinge for the remainder of this paper. The intrinsic SP splitting is not ubiquitous in NSTX. That is, in certain discharges, the 
non-axisymmetric magnetic perturbations generated by the intrinsic error fields may be canceled out by the background equilibrium fields. Or there may be a threshold of intrinsic error fields to produce intrinsic SP splitting.

While the pedestal pressure gradient increased by $\sim 30 \%$ after the application of $3-\mathrm{D}$ fields in case of no lithium coating $[12,13]$, which may be responsible for the change in ELM stability, there is no change observed in the pedestal pressure gradient by the applied 3-D fields in the case of lithium enhanced ELM-free H-mode discharges [16]. It is proposed that the ELM triggering by the application of 3-D fields is used to flush impurities during the long ELM-free, lithium-enhanced $\mathrm{H}$-mode discharges $[12,13]$. It is therefore important to understand heat and particle deposition mechanism of the 3-D field triggered ELMs onto the divertor surface.

Experimental setup and measurement techniques will be described in section 2 and the data analysis and interpretation will follow in section 3. Summary and conclusions will be discussed in section 4 .

\section{Experimental set-up and measurement technique}

The 3-D perturbation fields were generated with a set of six midplane coils, external but close-fitting to the vacuum vessel, that are typically used for error field correction and resistive wall mode feedback control $[17,18]$. The coils were configured to apply an $n=3$ field in the ELM-destabilization experiments, with a generated magnetic perturbation at the separatrix, $\delta \mathrm{B} / \mathrm{B}=0.6-0.7 \%$ for the peak $\delta \mathrm{B}$ at the coil centre and in the order of $0.1 \%$ for the integrated $\delta \mathrm{B}$ over the coil surface. The poloidal spectrum of the applied magnetic perturbation is broad at the plasma edge [13], reaching high enough mode numbers to be resonant with high edge safety factor values (q95 10).

The heat flux measurement is made with an SBF-161 infrared (IR) camera [19]. The reference point of the toroidal angle is located at the centre of the midplane coil \#1 and here the angle is measured counter-clockwise from the reference point. The IR camera is installed at the toroidal angle of $\varphi=135^{\circ}$ ( $225^{\circ}$ if clockwise). The camera takes IR images of the lower divertor plates in 2-D with a temporal resolution of 1.6 to $6.3 \mathrm{kHz}$ (depending on the frame size). The camera measures surface IR emission, which is converted to surface temperature from bench and in-situ calibrations. A 2-D heat conduction code called THEODOR [20] is used to calculate the divertor heat flux profile from the measured surface temperature. The calculation is carried out both in radial and tile depth directions for tiles with finite thickness as well as taking account of temperature dependent material parameters. However, the effect of Li coatings on the surface emissivity has not been properly assessed for the data to be presented in this paper and the condition of Li coated surfaces varies with plasma conditions, meaning that the surface 
temperature data can be different from the calibration by certain known amount. Therefore, the heat flux data computed from the surface temperature is not absolutely calibrated and the actual amount of heat carried by the heat flux profile is not known quantitatively. We thus rely on relative comparison of heat flux profiles for different experimental conditions. The several $\mathrm{kHz}$ framing rate enables heat flux measurement of transient events, such as ELMs and disruptions. The fast framing rate also facilitates measurement of the formation of striations in the divertor heat flux footprints, which can start to appear within 3-4ms after initiation of the 3-D field coil. The $\mathrm{D}_{\alpha}$ emission at the lower divertor target is recorded by a 1-D CCD camera installed at $\varphi=255^{\circ}\left(105^{\circ}\right.$ if clockwise). It is operated at $2 \mathrm{kHz}$ rate and with $\sim 0.5 \mathrm{~mm}$ spatial resolution and is a part of a system of CCD arrays [21]. The sheath-limited divertor plasma condition produced by Li enhanced plasmas presented in this paper makes the $\mathrm{D}_{\alpha}$ emission more closely correlated with the particle flux, because in this condition the $\mathrm{D}_{\alpha}$ emission comes more directly from the excitation of neutral particles which are the source of ionization to produce the particle flux. The derivation of particle flux from the $\mathrm{D}_{\alpha}$ measurement has been carried out in NSTX [22] and the recycling coeffcient with Li has been estimated from the SOLPS modeling [23] to be $\mathrm{R} \sim 0.92$, compared to $\mathrm{R} \sim 0.98$ without $\mathrm{Li}$. The electron temperature and density at the separatrix for these plasmas are normally $\mathrm{T}_{\mathrm{e}, \mathrm{sep}}=40-60 \mathrm{eV}, \mathrm{n}_{\mathrm{e}, \mathrm{sep}} \sim 1 \mathrm{e} 19 \mathrm{~m}^{-3}$. We therefore will use the measured $\mathrm{D}_{\alpha}$ profile as a proxy to the divertor particle flux profile for the rest of this paper.

\section{Data analysis and interpretation}

3.1 Formation of strike point splitting with the effect of 3-D field application

Figure 1 shows the temporal evolution of a lithium enhanced ELM-free H-mode discharge with an $n=3$ perturbation field applied. The $\mathrm{L}-\mathrm{H}$ transition is indicated by the drop of divertor $\mathrm{D}_{\alpha}$ emission at $\sim 110 \mathrm{~ms}$, and the H-mode was sustained until $\sim 570 \mathrm{~ms}$. The 3-D field perturbation was applied at $400 \mathrm{~ms}$ with constant amplitude of $0.75 \mathrm{kA}$. The $\mathrm{D}_{\alpha}$ trace shows that periodic Type-I ELMs were triggered after $\sim 45 \mathrm{~ms}$ following the application of 3 -D fields. The line-average electron density continued to rise in the H-mode phase even after the 3-D field application but the rate of increase is reduced due to the density purge by the ELMs triggered with the 3-D field, compared to the no ELM-triggering case with lower coil current of 0.5kA [16] where the rate of the density increase is not reduced even after the 3-D field application. The temporal and spatial evolution of the measured heat flux and $D_{\alpha}$ profiles are shown in figure 3 as a contour plot, for which each profile was measured at toroidal angles of $135^{\circ}$ and $255^{\circ}$, respectively. It is seen that the striation in both profiles is formed quite immediately, with a time constant consistent with field penetration time of $4-5 \mathrm{~ms}$, after the perturbation field application at 
400ms. This striation represents the split strike points with the effect of applied 3-D fields and the vacuum field line tracing reproduces the experimental observation quite well [16]. The inclusion of the plasma response inside the original separatrix by the IPEC calculation [24] is not found to affect the structure of split strike points significantly, ie the number and radial location of the generated lobes are unchanged by the inclusion of the plasma response. Here, the B-field generated by the plasma current up to a certain fraction of normalized flux ( $\left.e g, \Psi_{\mathrm{N}}=0.97\right)$ inside the separatrix is calculated by IPEC and is superposed to the vacuum field to begin field line tracing. Figure 2 shows puncture plots of magnetic footprints on the divertor target, one from a vacuum field line tracing code and the other from the IPEC calculation. They do not show the connection length profile comparison but are simply poloidal plane Poincare plots. It can be seen that the radial location and spacing of the generated lobes are little affected by the plasma response inside the separatrix. One should note that, however, this investigation has yet been carried out only for a limited set of plasma conditions and a more thorough examination for a wide range of plasma conditions is needed to draw a generalized conclusion. This result also needs to be compared with those from the resistive MHD approaches such as [25] in the future. The toroidal displacement of the IR and $D_{\alpha}$ cameras by $120^{\circ}$ is expected to produce $n=3$ periodicity in the divertor fluxes if the generated lobe structure is consistent with the imposed $n=3$ field structure. Indeed, the temporal and spatial evolution of striations is very similar for both heat flux and $\mathrm{D}_{\alpha}$ profiles (see figure 3). Note that the radial location of the original strike point moves back and forth by $2-3 \mathrm{~cm}$ in the period between $150 \mathrm{~ms}$ and $400 \mathrm{~ms}$. This is due to the plasma feedback control system working to keep the strike point position as constant as possible. In other words, the PF3 coil current is controlled to flow toward the direction of cancelling any radial movement of the equilibrium.

\subsection{Intrinsic strike point splitting in relation with intrinsic error fields}

It is also seen in figure 3 that the divertor flux profiles show certain degree of strike point splitting even before the application of external magnetic perturbation. The profiles show nearly monotonic decay, ie no strike point splitting, until $t \sim 190 \mathrm{~ms}$ and then begin to develop local peaks and valleys in the radial locations other than that of the original strike point at $\mathrm{r} \sim 35 \mathrm{~cm}$. This intrinsic strike point splitting is reflected by striations in the heat flux and $\mathrm{D}_{\alpha}$ profiles. The degree of splitting varies in time and both the heat flux and $\mathrm{D}_{\alpha}$ profiles show similar evolutions. Out of the possible sources of 3-D magnetic perturbation other than the external application, the intrinsic error field from the non-circularity of PF5 coil was modelled to be included in the vacuum field line tracing. It was recently shown [26] that PF5 produces primarily $\mathrm{n}=3$ error field and the $\mathrm{n}=3$ component $\left(B_{n=3}^{P F 5}=4 G\right)$ is approximately 4 times larger 
than the next highest component, which is $\mathrm{n}=2$ component $\left(B_{n=2}^{P F 5}=1 G\right)$. The inclusion of PF5 non-circularity in the vacuum field line tracing is therefore expected to produce dominant $n=3$ field structure although the model contains all non-circular components. Figure 4 shows comparison of computed connection length profile with the measured heat and particle flux profiles, both for the $\mathrm{n}=3$ application and the PF5 intrinsic error field cases. Here, the connection length is computed by following up a field line launched from the surface of outer target until it hits any PFC surface inside the vacuum vessel. It is seen that the radial location of local peaks agrees with each other very well. This indicates that intrinsic error fields may be one of the sources of the intrinsic SP splitting. However, it should be noted that the intrinsic SP splitting is not ubiquitous in NSTX. For some discharges, the intrinsic SP splitting is not observed during the whole plasma duration time in either the heat flux or $\mathrm{D}_{\alpha}$ profiles. Whether this is because the PF5 coil current in these discharges was too low $\left(\mathrm{I}_{\mathrm{PF} 5}=5.6 \mathrm{kA}\right)$ to produce SP splitting, compared to the other case $\left(\mathrm{I}_{\mathrm{PF} 5}=7.5 \mathrm{kA}\right)$, or the 2-D equilibrium fields superposed by the 3-D perturbation fields reacted toward the direction of canceling 3-D field effect is not clear at present.

\subsection{Heat flux deposition during the ELMs triggered by 3-D field application}

The externally imposed 3-D fields trigger ELMs and the strong heat and particle expulsion by the triggered ELMs onto the divertor surface is indicated as several vertical lines in figure 3. The frame speed of the IR camera was sufficiently high $(\sim 3.8 \mathrm{kHz})$ to measure heat flux profiles during the ELM. Figure 5 shows the heat flux profiles at the ELM peak and immediately $(0.6 \mathrm{~ms})$ before the ELM occurrence. One can notice that the SP splitting is persistent even during the ELM ( $\mathrm{t}=444.6 \mathrm{~ms})$ as the profile exhibits local peaks and valleys. Also, the radial location of the split strike points before and during the ELM agrees with each other very well. This indicates that the heat flux profile from ELMs triggered by $n=3$ fields may follow imposed field structure, ie 3-D field triggered ELMs appear to be phase locked to the externally applied perturbation. This has an important implication that the distribution of divertor heat flux of the triggered ELMs can be aligned with the footprint pattern generated by the imposed 3-D field.

\section{Summary and conclusions}

The non-axisymmetric perturbation fields, either externally imposed or internally arisen, are observed to cause the splitting of strike point in NSTX H-mode plasmas. The applied 3-D fields modify the heat and particle flux profiles in a manner consistent with vacuum field line tracing. The inclusion of plasma response inside the separatrix in the field line tracing does not 
significantly change the radial location and spacing of split strike points. This is important because it indicates that vacuum field line tracing may be sufficient to predict the structure of the generated strike point splitting. The intrinsic strike point splitting observed in both profiles for some NSTX discharges is thought to be related to the intrinsic error fields in PF5 coil. The noncircularity of PF5 coil was modelled to be included in the vacuum field line tracing and was shown to generate very similar field structure to the case of $n=3$ field application. This indicates that the intrinsic error field may be one of the sources to produce intrinsic strike point splitting. However, there are also discharges with no intrinsic strike point splitting for the whole duration of the discharge. It is not clear at present what is causing the difference between these two cases. It is possible that the current of PF5 coils was not sufficiently high to induce the strike point splitting, or the background 2-D equilibrium fields for those discharges reacted toward the direction of cancelling out the effect of superposed 3-D fields. A detailed investigation to answer this question is needed. Heat flux profiles measured at the peak of triggered ELMs show that the lobe structure is persistent even during the ELMs and the heat flux follows imposed field structure, implying that the ELMs may be phase-locked to the externally imposed perturbation.

\section{Acknowledgements}

This work was funded by the US Department of Energy, contract numbers DE-AC05000R22725, DE-AC52-07NA27344(LLNL), and DE-AC02-09CH11466.

\section{References}

[1] O. Schmitz, T. E. Evans, M. E. Fenstermacher, et al, Plasma Phys. Control. Fusion 50 (2008), 124029

[2] M. J. Schaffer, J. E. Menard, M. P. Aldan, J. M. Bialek, T. E. Evans and R. A. Moyer, Nucl. Fusion 48 (2008), 024004

[3] O. Schmitz, T. E. Evans, J. Boedo, et al, These precedings

[4] S. Jachmich, G. Arnoux, S. Brezinsek et al, These precedings

[5] M. W. Jakubowski, T. E. Evans, C. J. Lasnier, et al, These precedings

[6] A. Kirk, E. Nardon, R. Akers, et al, Nucl. Fusion 50 (2010) 034008

[7] J. L. Luxton, Nucl. Fusion 42 (2002), 614-33

[8] T. E. Evans, R. A. Moyer, K. H. Burrell, et al, Nature Phys. 2 (2006) 419-23

[9] T. E. Evans, M. E. Fenstermacher, R. A. Moyer, et al, Nucl. Fusion 48 (2008) 024002

[10] M. Ono, S. M. Kaye, Y.-K. M. Peng, et al, Nucl. Fusion 40 (2000) 557

[11] R. Maingi, T. H. Osborne, B. P. LeBlanc, et al, Phys. Rev. Lett. 103 (2009) 075001

[12] J. M. Canik, R. Maingi, T. E. Evans, et al, Phys. Rev. Letts. 104 (2010) 045001

[13] J. M. Canik, R. Maingi, T. E. Evans, et al, Nucl. Fusion 50 (2010) 034012

[14] T. E. Evans, R. K. W. Roeder, J. A. Carter, B. I. Rapoport, M. E. Fenstermacher and C. J. Lasnier, J. Phys : Conf. Ser. 7 (2005) 174-90 
[15] M. W. Jakubowski, T. E. Evans, M. E. Fenstermacher, et al, Nucl. Fusion 49 (2009) 095013

[16] J-W. Ahn, J. M. Canik, R. Maingi, et al, Nucl. Fusion 50 (2010), 045010

[17] S. A. Sabbagh, R. E. Bell, J. E. Menard, et al, Phys. Rev. Lett. 97 (2006) 045004

[18] J. E. Menard, M. G. Bell, R. E. Bell, et el, Nucl. Fusion 47 (2007) S645

[19] J-W. Ahn, R. Maingi, D. Mastrovito, and A. L. Roquemore, Rev. Sci. Instrum. 81 (2010) 023501

[20] A. Herrmann, W. Junker, K. Gunther, et al, Plasma Phys. Control. Fusion 37 (1995) 17

[21] V. A. Soukhanovskii, A. L. Roquemore, C. H. Skinner, et al, Rev. Sci. Instrum. 74 (2003) 2094

[22] V. A. Soukhanovskii, R. E. Bell, C. Bush, et al, J. Nucl. Maters 390-391 (2009) 516-519

[23] J. M. Canik, et al, These precedings

[24] J.-K. Park, , A. H. Boozer, and A. H. Glasser, et al, Phys. Plasmas 14 (2007) 052110

[25] E. Nardon, P. Cahyna, E. De La Luna, et al, These precedings

[26] J. E. Menard, R. E. Bell, D. A. Gates, et al, Nucl. Fusion 50 (2010) 045008 


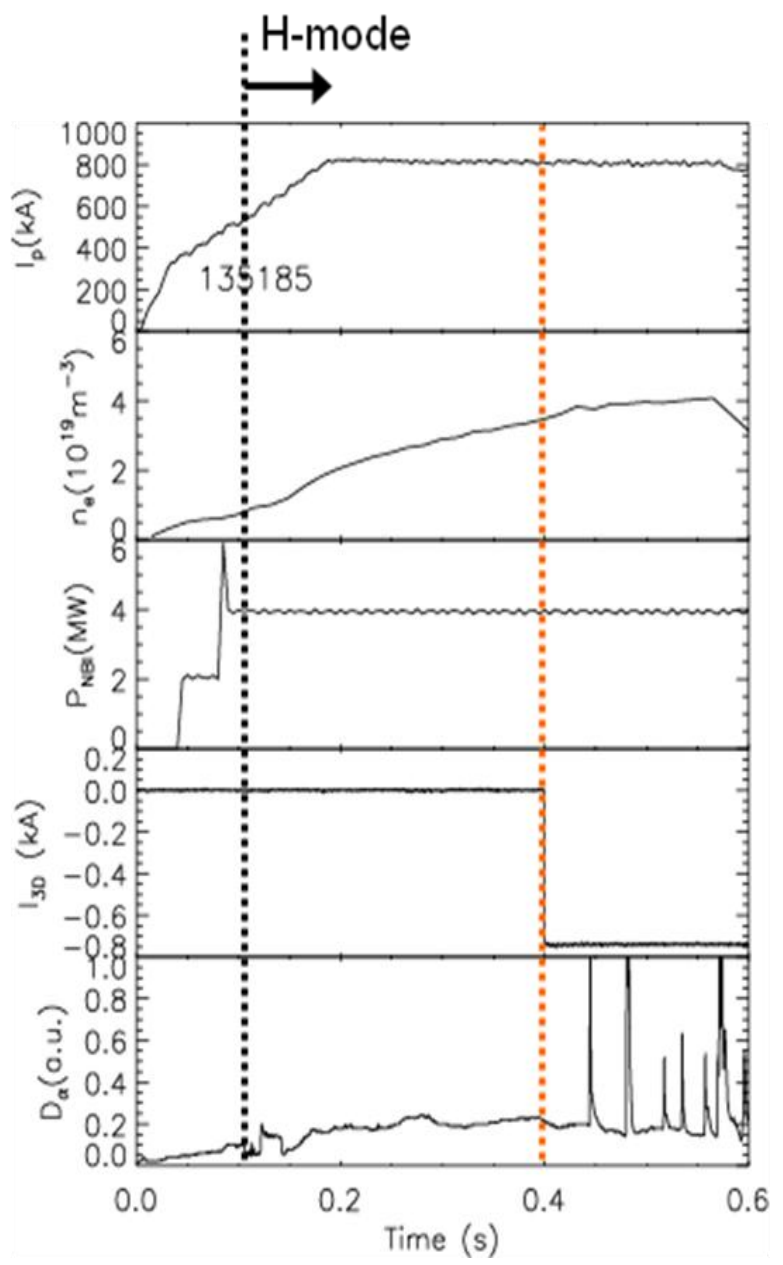

Figure 1. Time evolution of various discharge parameters for a 3-D field applied shot 135185: (a) plasma current, (b) line averaged density, (c) injected NBI power, (d) total stored energy, (e) current in the external 3-D coil, (f) $\mathrm{D}_{\alpha}$ signal for lower divertor. Note that the 3-D field coil was switched on at $400 \mathrm{~ms}$ to $-0.75 \mathrm{kA}$ and this triggered ELMs in about $\sim 45 \mathrm{~ms}$ after the onset of $n=3$ perturbation field at $t=400 \mathrm{~ms}$ 


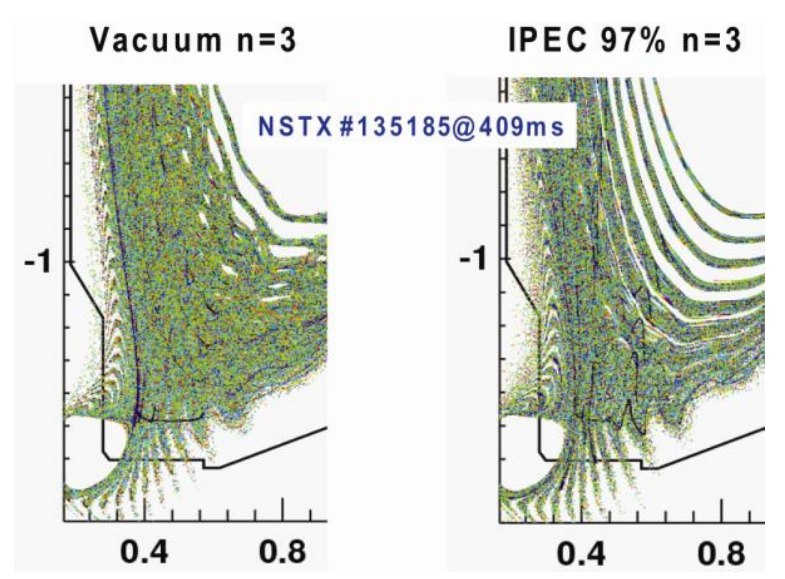

Figure 2. Poloidal Poincare plot from a vacuum field line tracing calculation (left) and from the one with the plasma response included in the field line tracing, up to $97 \%$ of normalized flux ( $\left.e g, \Psi_{\mathrm{N}}=0.97\right)$ inside the separatrix, calculated by IPEC (right) 

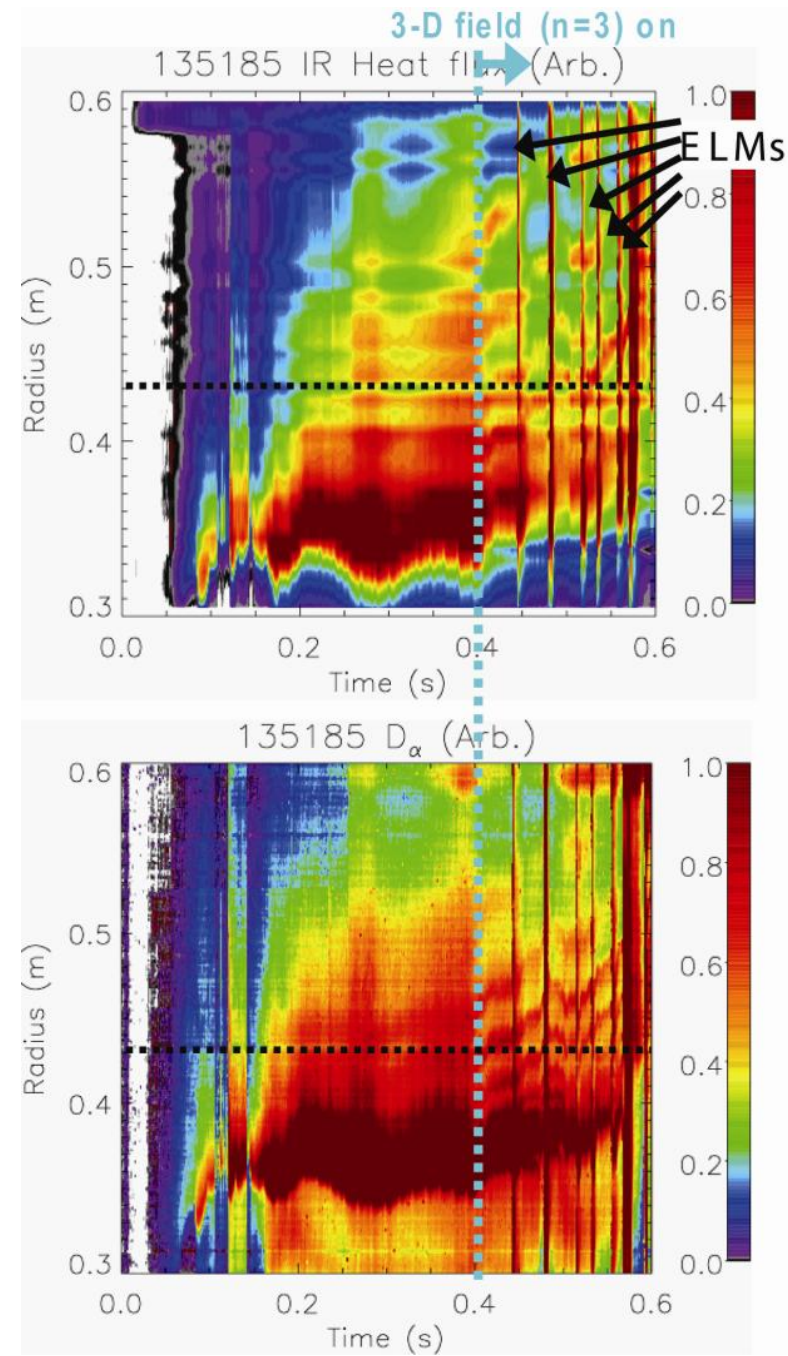

Figure 3. Contour plot of measured heat flux (upper) and $\mathrm{D}_{\alpha}$ (lower) profiles with $\mathrm{n}=3$ perturbation field applied from $\mathrm{t}=400 \mathrm{~ms}$. Triggered ELMs are indicated by the vertical lines in both contour plots. Note that a tile gap ( $2 \mathrm{~mm}$ wide) is located at $\mathrm{r}=43 \mathrm{~cm}$, which is represented by the black dotted line. 

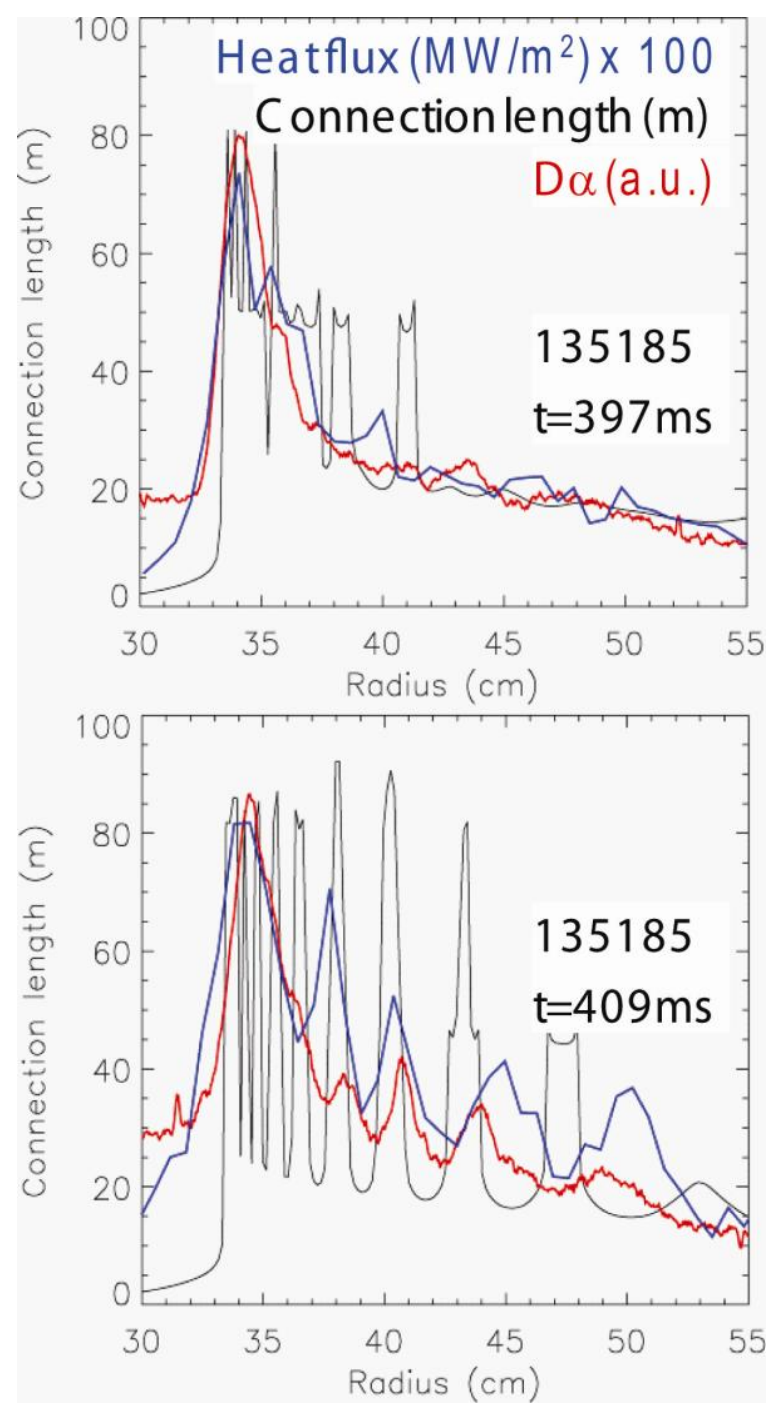

Figure 4. Comparison of computed connection length profile by the vacuum field line tracing (black) with heat flux (blue) and $\mathrm{D}_{\alpha}$ (red) profiles. The upper plots are for the PF5 intrinsic error field case and the lower ones are for the $\mathrm{n}=3$ field applied case. 


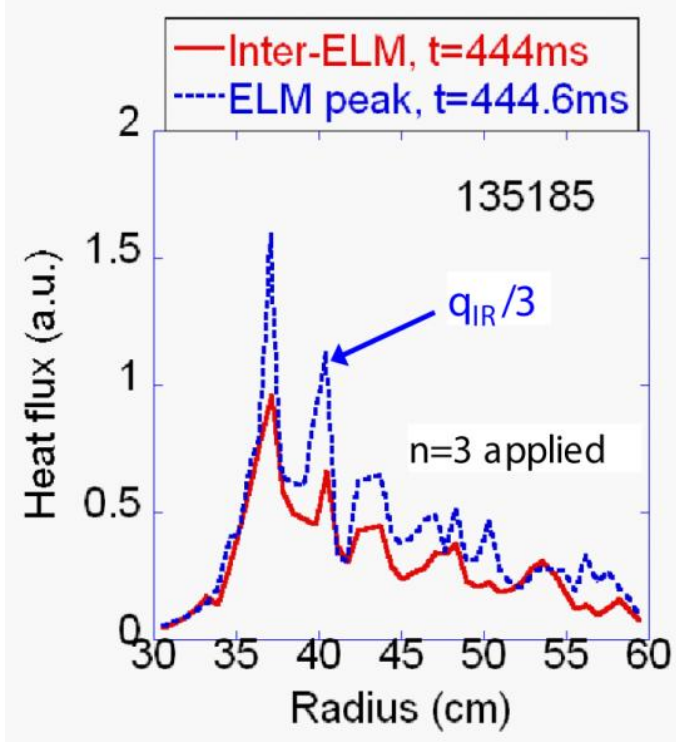

Figure 5. Heat flux profiles measured at the ELM peak (blue dotted line), divided by 3 , and during the inter-ELM period, $0.6 \mathrm{~ms}$ before the peak (red solid line) 